\title{
Design and Virtual Simulation of an Optimal PID/LQRT-PSO ControlSystem for 2WD Mobile Robots
}

\author{
Markov Djoumessi Mbihi ${ }^{(1)^{*}}$, Bertrand Lonla Moffo ${ }^{(2)}$, Léandre Nneme Nneme ${ }^{(3)}$ \\ (1),(2, (3) Laboratory of Computer Science Engineering and Automation, \\ ENSET, University of Douala, Cameroon \\ mbihimarkov@yahoo.fr
}

\begin{abstract}
This research paper presents a relevant contribution to optimal control of angular velocities with trajectories tracking for2WD mobile robots. The dynamic model of each robotic servomechanisms is modeled from experimental data as a second order transfer function. Then, the direct and reverse kinematic equations are analytically developed. Then, the LQRT (Linear Quadratic Regulator with Tracking) gains for angular velocity of servo-mechanisms, are computed over infinite time horizon from corresponding Riccatti equations, using PSO (Particles Swarm Optimization) values of weighted matrix $Q$ and $R$. Therefore, the LQRT solution computed from PSO of $Q$ and $R$ is known as LQRT-PSO. In addition, the LQRT-PSO gains computed from the related Riccati equations are transformed into equivalent PID/LQRT-PSO gains for angular velocities control. Furthermore, a trajectory tracking flowchart is designed to reinforce the robustness of the overall control system. On the other hand, relevant developments conducted in the design step, are organized into an overall Matlab/Simulink PID/LQRT-PSO scheme. Finally, the obtained simulation results are presented in order to show the high performance of the proposed PID/LQRT-PSO control scheme for 2WD mobile robots.
\end{abstract}

Keywords: 2WD mobile robot, design PID/LQRT, PSO, PID/LQRT-PSO, trajectory tracking flowchart, virtual simulation.

\section{INTRODUCTION}

A wide variety of design methods of optimal controllers for robotic control systems, are encountered in the literature. The first class consists of computing strategies for optimal PID regulators, e.g., ISE (Integral Square Error), IAE (Integral Absolute Error), ITAE (Integral Time Absolute Error), ITSE (Integral Time Square Error), and more [1], [2]. They are available as ready-to-use formulas. However, they are mainly addressed to firstorder dynamic processes with input delay. Then, the second class of optimal design methods for robotic control systems is known as PSO (Particles swam optimization)[3]-[6]. Although more accurate, their practical design requires greedy numerical analysis tasks. Furthermore, the third class of optimal controllers for robotic automation, isa LQR (linear quadratic regulator)[7]-[12]. Fortunately, a LQR is available as a ready-touse package in Matlab/Simulink, for rapid computing of LQR gains, at least over an infinite optimization time. However, the synthesis of a $L Q R$ requires numerical values of weighting matrix $Q$ and $R$. Usually, values of $Q$ and $R$ are chosen according to a TEC (Trial and Error Computing) technique. In which case, the optimality of the resulting optimal LQR control might not be effective. The fourth class of optimal control design method is the LQR-PSO algorithm [13]-[16]. In this case, a prior optimization of weighting matrix $Q$ and $R$ is conducted. Then, the overall characteristics of the resulting LQR can be significantly improved.

However, although LQR control systems are relevant in virtual design and simulation contexts, its real time implementation might become very intricate. Indeed, the real time implementation of a LQR for an-order dynamic process, requires the availability of $n$ appropriate sensors[17], i.e. one sensor per state. As an example, in the case of a $2 \mathrm{WD}$ mobile robot with third order dynamics, a set of three physical sensors are required for state feedback control. In addition, an 
additional potential problem which might occur in practice is that, a few states of the whole robotic control system can be fictitious without meaningful physical quantity. In such cases, the required sensors cannot be available in the market and cannot be easily built. Of course, it is possible to overcome the lack of physical sensors by optimal state observers. Unfortunately, the whole LQR control system with $\mathrm{n}$ states, and the related $n$-order observer, might computationally become very tedious and intractable in a real time context.

These potential drawbacks have motivated a new design approach of optimal control systems, known as PID/LQR controls. They combine the design simplicity of standard LQR technique, as well as a great robustness and low cost implementation cost of PID controllers. The PID/LQR design approach has been successfully used in[18]-[23], for many application areas, including optimal PID/LQR control of Buck converters[12],[23]. These works have motivated our ambition to study in this paper, the feasibility and qualities of a pioneering PID/LQRT-PSO control system for mobile robots. Without lost of generality, the great emphasis on this paper is more on 2WD mobile robot.

Section 2 of this paper deals with the research methodology and tools, used for the development of optimal PID/LQRT-PSO control systems for 2WD robots. Then, in Section 3 , virtual simulation results obtained are presented and discussed. Finally, the paper is concluded in Section 4.

\section{LQRT-TEC AND LQRT-PSO DESIGN ALGORITMS FOR 2WD ROBOT}

\subsection{Dynamic Modeling of a 2WD robot}

The dynamic system used in this paper, is a 2WD mobile robot with two independent DC servo-mechanisms as illustrated in Figure 1. Because of the unavailability of the dynamic model associated with the 2WD robot, it will be developed using experimental modeling approach, i.e., on the basis of real data acquired from tests and measurements[24]. Since the 2WD robot consists of 02 equivalent servomechanisms, (e.g. servomotor with gear for each case), the following assumptions are used to develop a whole decoupling dynamic model.

- Both servomechanisms have identical electrical/mechanical characteristics;
- Both gear motors are submitted to rotational and charge constraints during robot motion;

- Experimental modeling error can be rigorously cancelled under robust feedback control conditions.

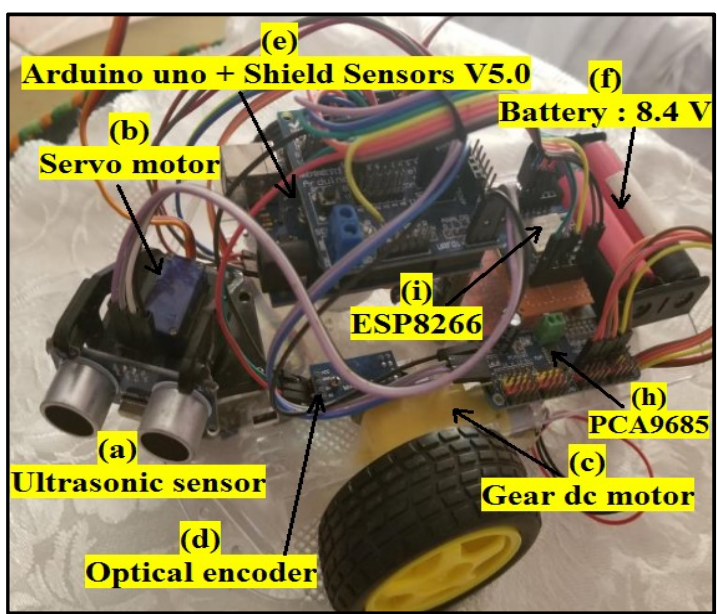

Fig. 1. 2WD mobile robot

In addition, the experimental models of both servomechanisms are similar, whereas the logistic materials used to obtain experimental data are given as follow:

- 02 similar servomechanisms (DC motor with gear);

- 02 optical encoders, each for single servomechanism;

- 02encoder disks with 20 slots, each for single servomechanism;

- Arduino Uno microcontroller;

- $\quad 8.4$ V Lithium Polymer Battery.

As observed on Figure 1, the angular velocity data of the gear motor (in rad/s), was acquired during 62 seconds and saved for next step. Then, the processing of experimental data using MATLAB system Identification Toolbox, with $91.27 \%$ estimation, led to the estimated transfer function eq.(1).

$$
\begin{aligned}
& G(s)_{O L}=\frac{41.83}{s^{2}+8.699 s+12.91} \\
&=\frac{K_{s} \omega_{n}^{2}}{s^{2}+2 \xi \omega_{n} s+\omega_{n}^{2}} \\
& K_{s}=\frac{41.83}{12.91}, \omega_{n}=\sqrt{12.91}, \quad \xi=\frac{8.699}{2 \omega_{n}}
\end{aligned}
$$

Where $G(s)_{O L}$ is the estimated open loop transfer function of one servomechanism. 


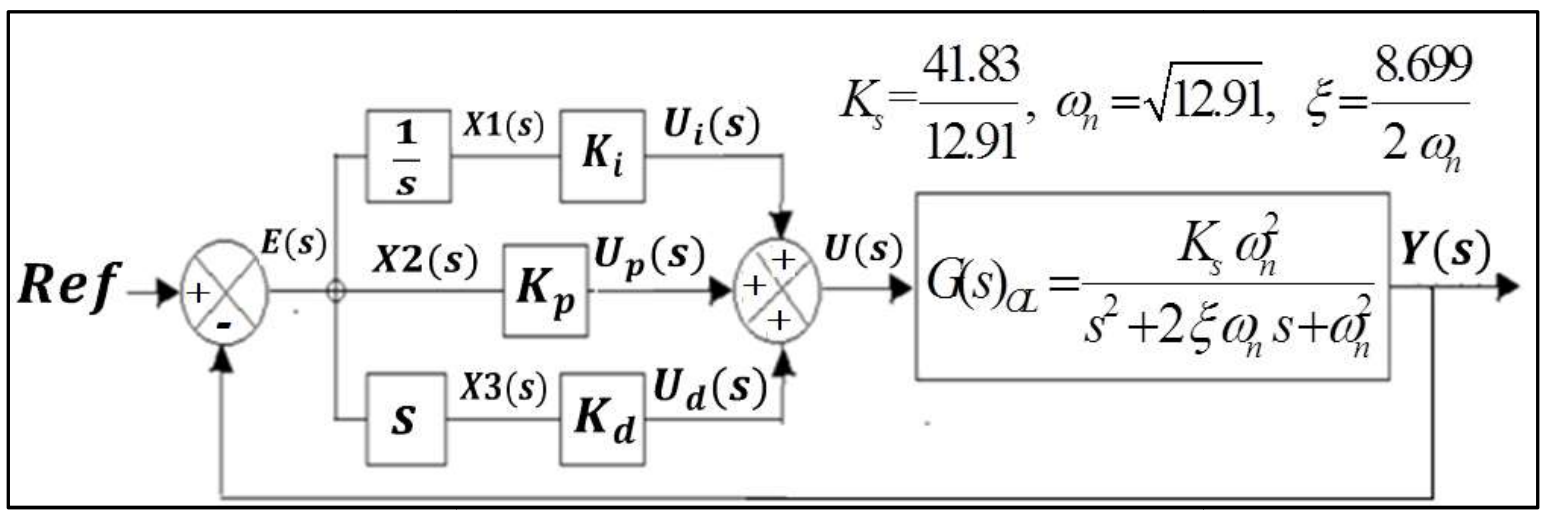

Fig. 2 Full PID/LQRTcontrol scheme with arbitrary choice of states $x 1, x 2$ and $x 3$.

\subsection{LQRT-TEC design algorithm}

In Figure 2, a full PID/LQRT control scheme for an open loop $2^{\text {nd }}$ order process (1), is presented. Unlike a simple PID/LQR policy, it resort to an augmented state, e.g. $\mathrm{xi}=\mathrm{x} 1$, for the sake of better tracking of set trajectories if any. As a benefit, it behaves under feedback of three states $\{x 1, x 2, x 3\}$, as a robust PID control of a second order dynamic system.

However, optimal PID control parameters computed from equivalent LQRT controller by TEC method of weighing matrices $R$ and $Q$, are called PID-LQRT strategies. In section 2.3, a more powerful PID/LQRT-PSO algorithm, founded ona prior optimization of $R$ and $Q$ values, is initiated and developed.

A state space realization of the open loop transfer function in eq.(1), computed using Matlab ss command, is given by eq.(2)

$$
\begin{aligned}
& \left\{\begin{array}{l}
\square=A x+B u \\
y=C x+D u
\end{array}\right. \\
& \begin{cases}A=\left[\begin{array}{cc}
-8.699 & -3.228 \\
4.0 & 0
\end{array}\right], & B=\left[\begin{array}{l}
4 \\
0
\end{array}\right] \\
C=\left[\begin{array}{ll}
0 & 2.614
\end{array}\right], & D=0\end{cases}
\end{aligned}
$$

Therefore, $x, y$ and $u$ within eq.(2) are state vector, physical output and servomotor control voltage, respectively. Furthermore, expression (3) stands for augmented state dynamics, with integral state error $x i$ for trajectories tracking purposes.

$$
\begin{aligned}
& \left\{\begin{array}{l}
\square \\
x \\
\square \\
x_{i}
\end{array}\right]=A e q\left[\begin{array}{l}
x \\
x_{i}
\end{array}\right]+B e q u-\left[\begin{array}{c}
0 \\
0 \\
y r e f
\end{array}\right] \\
& y=C e q\left[\begin{array}{l}
x \\
x_{i}
\end{array}\right] \\
& \text { Aeq }=\left[\begin{array}{ll}
A & 0 \\
C & 0
\end{array}\right], B e q=\left[\begin{array}{l}
B \\
0
\end{array}\right] \\
& \text { Ceq }=\left[\begin{array}{ll}
C & 0
\end{array}\right]
\end{aligned}
$$

In (3), $y_{\text {ref }}=$ angular velocity set-point, and $x_{i}=$ integral state variable.

Before tuning a control law foreq.(3), it's necessary to check the full rank of its controllability Matrix M.A direct computing using Matlab ctrb command $M=c t r b(A e q$, $B e q)$, leads to (4):

$$
M=\left[\begin{array}{ccc}
4.0000 & -34.7960 & 251.0424 \\
0 & 16.0000 & -139.1840 \\
0 & 0 & 41.8240
\end{array}\right]
$$

Then, the rank of $\mathrm{M}$ computed using Matlab command $\operatorname{rank}(M)$ is equal to size of $A$ eq matrix, i.e, equal to 3 . Hence, the augmented dynamic state model in eq.(3) is fully controllable, i.e., the existence of state feedback gain $K_{L Q R T}$ that minimizes the cost function in eq.(5), is proven.

$$
J=\int x^{T}(t) Q x(t)+u^{T}(t) R u(t)
$$

In (5), $Q$ and $R$ are augmented symmetric weighting matrix, with positive semi-definite 
and positive definite properties respectively. As well known in optimal control theory literature, optimizing eq.(5) given state constraint eq.(3), lead to Riccatti Algebraic eq.(6),

$$
A^{T} P+P A-P B R^{-1} B^{T} P+Q=0
$$

Where $P^{*}$ is the unknown positive definite symmetric matrix to be computed. Obviously, solving eq.(6) requires a prior knowledge of $Q$ and $R$ weighting matrix. Subsequently, if values of $Q$ and $R$ are preliminary chosen using TEC (Trial and Error Computing) method, then $P^{*}=P_{T E C}$, the resulting optimal state feedback control gain is given by eq.(7).

$$
K_{T E C}=R^{-1} B^{T} P_{T E C}
$$

\subsection{LQRT-PSO design algorithm}

The PSO was formulated by J. Kennedy and R. Eberhart in 1995. It is based on swarm behavior such as fish and bird schooling in nature, and may have some similarities with genetic algorithms, but it is much simpler because it does not use mutation/crossover operators.

Instead, it uses the real-number randomness and the global communication among the swarm particles[25]. Each particle moves on the cost surface at a given velocity. The particles update their velocities and positions based on the local and global best solution in each iteration according to eq.(8).

$$
\left\{\begin{array}{l}
v_{i j}(t+1)=\gamma v_{i j}(t)+c_{1} r_{1}\left(p_{i j}-x_{i j}(t)\right) \cdots \\
\cdots+c_{2} r_{2}\left(g_{j}-x_{i j}(t)\right) \\
x_{i j}(t+1)=x_{i j}(t)+v_{i j}(t+1)
\end{array}\right.
$$

The relevant notations used in the PSO algorithm are defined as follows:

$$
\begin{aligned}
& i=\{1,2, \ldots, n\} ; \\
& j=\{1,2, \ldots, d\} ; \\
& n \text {-Number of particles; } \\
& d \text { - Number of dimension; } \\
& \gamma \text {-Inertial weight factor; } \\
& c_{1}, c_{2}-\text { Coefficients acceleration with } \\
& c_{1}+c_{2} \leq 4 ;
\end{aligned}
$$

$r_{1}, r_{2}$-Independent uniform random numbers within interval $\left[\begin{array}{ll}0 & 1\end{array}\right]$;

$v_{i j}$ - Particles velocity;

$x_{i j}$ - Position velocity;

$p_{i j}$ - Best local solution;

$g_{j}-$ Best global solution;

$t$-Pseudo time of iteration counter;

The best weighting matrix $Q_{P S O}$ and $R_{P S O}$ to be computed by PSO algorithm, depends on a given target cost function. In this paper, we use the cost function described by eq.(9) given eq.(10).

$$
\begin{aligned}
& J=\min \left\{\left|\sum \cos (V, W)\right|\right\} \cdots \\
& \cdots+\min \left\{\left|\frac{y_{\text {measured }}(t)-y_{\text {desired }}(t)}{y_{\text {desired }}(t)}\right|\right\}
\end{aligned}
$$

With

$$
\cos (V, W)=\frac{\left|W_{i}^{T} V_{i}\right|}{\left\|W_{i}\right\|_{2}\left\|V_{i}\right\|_{2}}
$$

Where:

$W_{i}$ - Is the left eigenvector;

$V_{i}$ - Is the right eigenvector;

$\|\bullet\|_{2}$ - Is the $\mathrm{L}_{2}$-Norm operator.

$y_{\text {desired }}(t)-$ Is desired output;

$y_{\text {measured }}(t)-$ Is measured output;

For the sake of better understanding, Table 1 provides a similarity technical dictionary for LQRT-PSO and LQRT-TEC algorithms, whereas the PSO flowchart is presented in Figure 3. 
Table 1: Key words equivalence of LQRTPSO and LQRT-TEC

\begin{tabular}{|c|c|c|}
\hline No & $\begin{array}{c}\text { LQRT-PSO } \\
\text { key words }\end{array}$ & $\begin{array}{c}\text { LQRT-TEC } \\
\text { key words } \\
\text { Trial solutionof } \\
\text { field of states } \\
\text { variables }\end{array}$ \\
\hline 1 & Particles Swarm & $\begin{array}{c}\text { Number of } \\
\text { elements of } \mathbf{Q} \\
\text { and } \mathbf{R}:\end{array}$ \\
\hline 2 & $\begin{array}{c}\text { dimensions } \\
d\end{array}$ & \\
\hline
\end{tabular}

\begin{tabular}{|c|c|c|}
\hline & & $\mathbf{q 1 , q 2 , q 3}$ and $\boldsymbol{r}$ \\
\hline 3 & Fitness function & Cost function \\
\hline 4 & $\begin{array}{c}\text { Number of } \\
\text { particles }\end{array}$ & $\begin{array}{c}\text { Number of } \\
\text { states variables }\end{array}$ \\
\hline 5 & Qpso and Rpso & Qtec and Rtec \\
\hline 6 & Ppso and Kpso & Ptec and Ktec \\
\hline
\end{tabular}

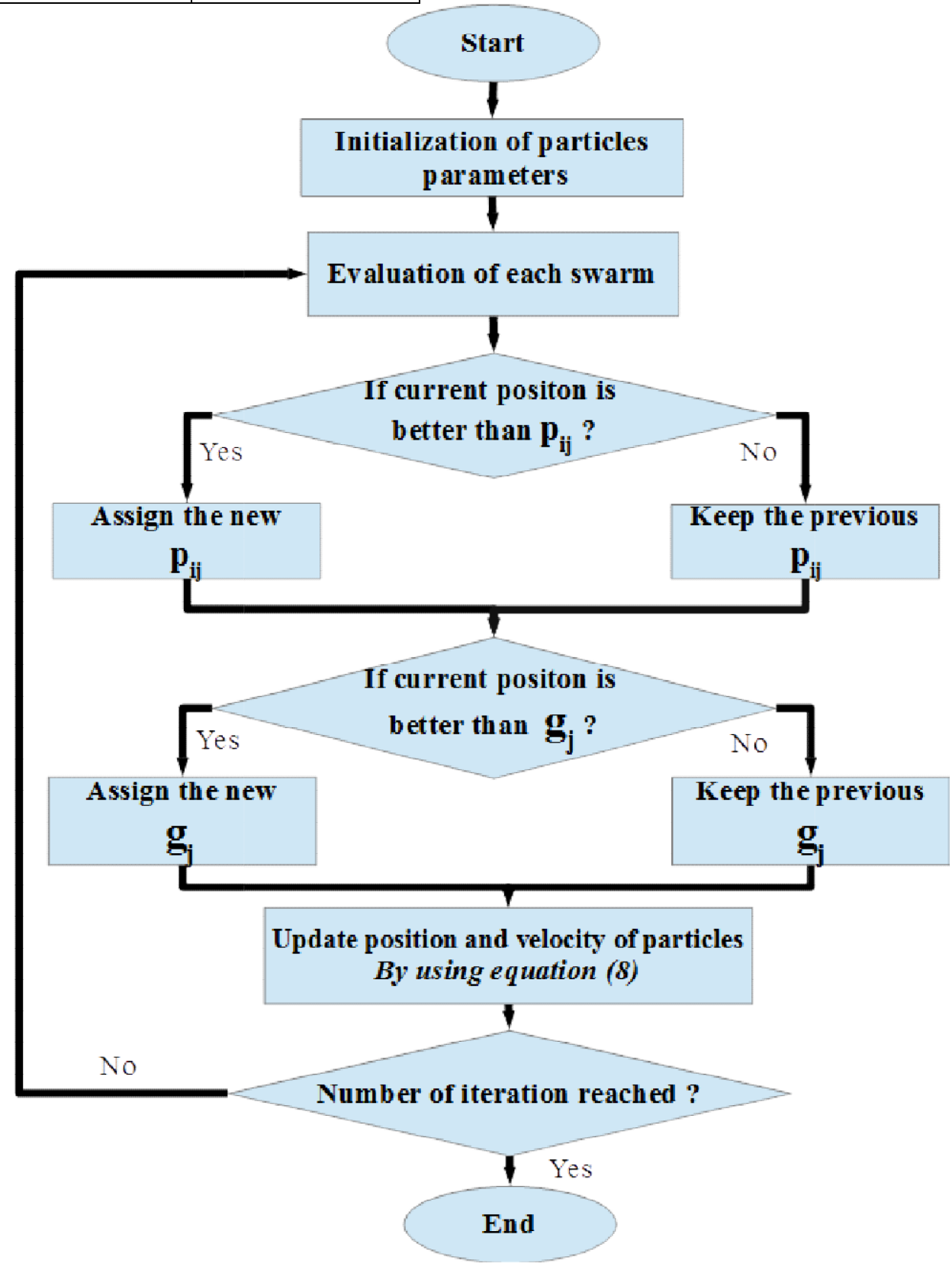

Fig.3.PSO flowchart

Compared to eq.(7), the optimal solution $\mathrm{P}^{*}=$ the corresponding state feedback gain $\mathrm{K}_{\mathrm{PSO}}$, $P_{P S O}$, computed from $P S O$ of $Q$ and $R$ and are linked by relationship eq.(11). 


$$
K_{P S O}=R^{-1} B^{T} P \text { PSO }
$$

It's important to clarify some terms in the flowchart in Figure 3, such as particles, which are random solutions generated by normal uniform law taken in a field of potential solution, described by swarm. Finally, the number of iteration mentioned in Figure 3 , is number of next generation of solution computed to reach to a permanent convergence zone.

\section{4. $P I D / L Q R T-P S O$ design}

In order to avoid high costs calculation, and to reduce complexity in the realization of optimal controllers, a LQRT/PID method is developed for the linear systems. It is the case of Calovic and Cuk [18] in their paper on multivariable linear systems. But it was criticized by Subrata in its paper [19].

In this paper, we use the same principle applied in[12] and [23] for optimal regulation of angular velocity of one of DC motor gear of a 2WD mobile robot. The controllability conditions being already satisfied, the optimal control can be written:

$$
u^{*}=-R^{-1} B^{T} P_{L Q R-P S O} x
$$

We define:

$$
\tilde{K}_{p}=R^{-1} B^{T} P_{11}
$$

And

$$
\tilde{K}_{i}=R^{-1} B^{T} P_{12}
$$

With

$$
\begin{aligned}
& P_{11}=P_{L Q R-P S O}(1: f r, 1: f r) \text { (15) } \\
& P_{12}=P_{L Q R-P S O}(1: f r, f r+1: f r+
\end{aligned}
$$

Where $f r$ is the length of the first row of state matrix $A_{\text {eq }}$, while $f c$ is the length of the first column of observation matrix $C_{e q}$, both $A_{e q}$, and Ceq being earlier given in eq.(2).

Given $\tilde{K}_{p}$ and $\tilde{K}_{i}$, then the corresponding optimal PID parameters $K_{p}, K_{d}$ and $K_{i}$ can be computed according to eq.(17) and eq.(18) respectively.

$$
K_{i}=\left(I_{m}+K_{d} C B\right) \tilde{K}_{i}
$$

$\left[\begin{array}{ll}K_{p} & K_{d}\end{array}\right]=\tilde{K}_{p} \bar{C}^{-1}$

With

$$
\bar{C}=\left[\begin{array}{c}
C \\
C A-C B \tilde{K}_{p}
\end{array}\right]
$$

Where $I_{m}$ is the identity matrix

\subsection{Kinematic model of $2 W D$ robot}

The kinematic model of a 2WD robot schematized in Figure 4, is developed under a few assumptions are called from [26]:

- No slip between the wheel and the ground;

- The vehicle cannot have lateral moving in order to maintain the constraint non-holonomic;

- The motion of the mobile robot is limited to $X Y$ plane.

From figure4, equation (19) is obvious.

$$
\left\{\begin{array}{l}
\dot{x_{a}}=v \cdot \cos \theta \\
\dot{y_{a}}=v \cdot \sin \theta
\end{array}\right.
$$

In addition, the linear and angular velocitiessimultaneously evolves according tothe set of eq.(20) andeq.(21).

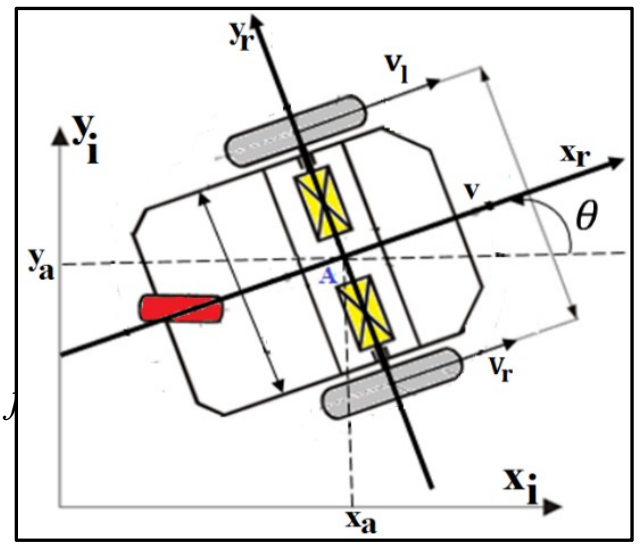

Fig. 4. Kinematic diagram of a $2 W D$ robot

$$
\begin{gathered}
v=\frac{v_{l}+v_{r}}{2}=R \frac{\omega_{r}+\omega_{l}}{2} \\
\omega=\theta=R \frac{\omega_{r}-\omega_{l}}{2 L}
\end{gathered}
$$

Where:

$v$ - The linear velocity $(\mathrm{cm} / \mathrm{s})$; 
$R$ - The wheel radius $(\mathrm{cm})$;

$\omega$ - The angular velocity $(r d / s)$;

$L$ - Distance between center of mass and one wheel $(\mathrm{cm})$;

$v_{l}, \omega_{l}-$ Left linear and angular velocity;

$v_{r}, \omega_{r}-$ Right linear and angular velocity;

The reverse kinematic model eq.(22) and eq.(23), is computed using eq.(20) and eq.(21).

$$
\omega_{r}=\frac{v+L \omega}{R}
$$

$$
\omega_{l}=\frac{v-L \omega}{R}
$$

\subsection{Proposed optimal control system}

The pioneering Simulink-based virtual control scheme, developed in this paper for 2WD robots, is presented in Figure 5.The subsystems (d) and (b) are drawn from the set of $\{$ eq.(20), eq.(21)\} and \{eq.(22), eq.(23)\} respectively. The subsystem (a) implements the tracking algorithm, according to the state flowchart illustrated in Figure 6.

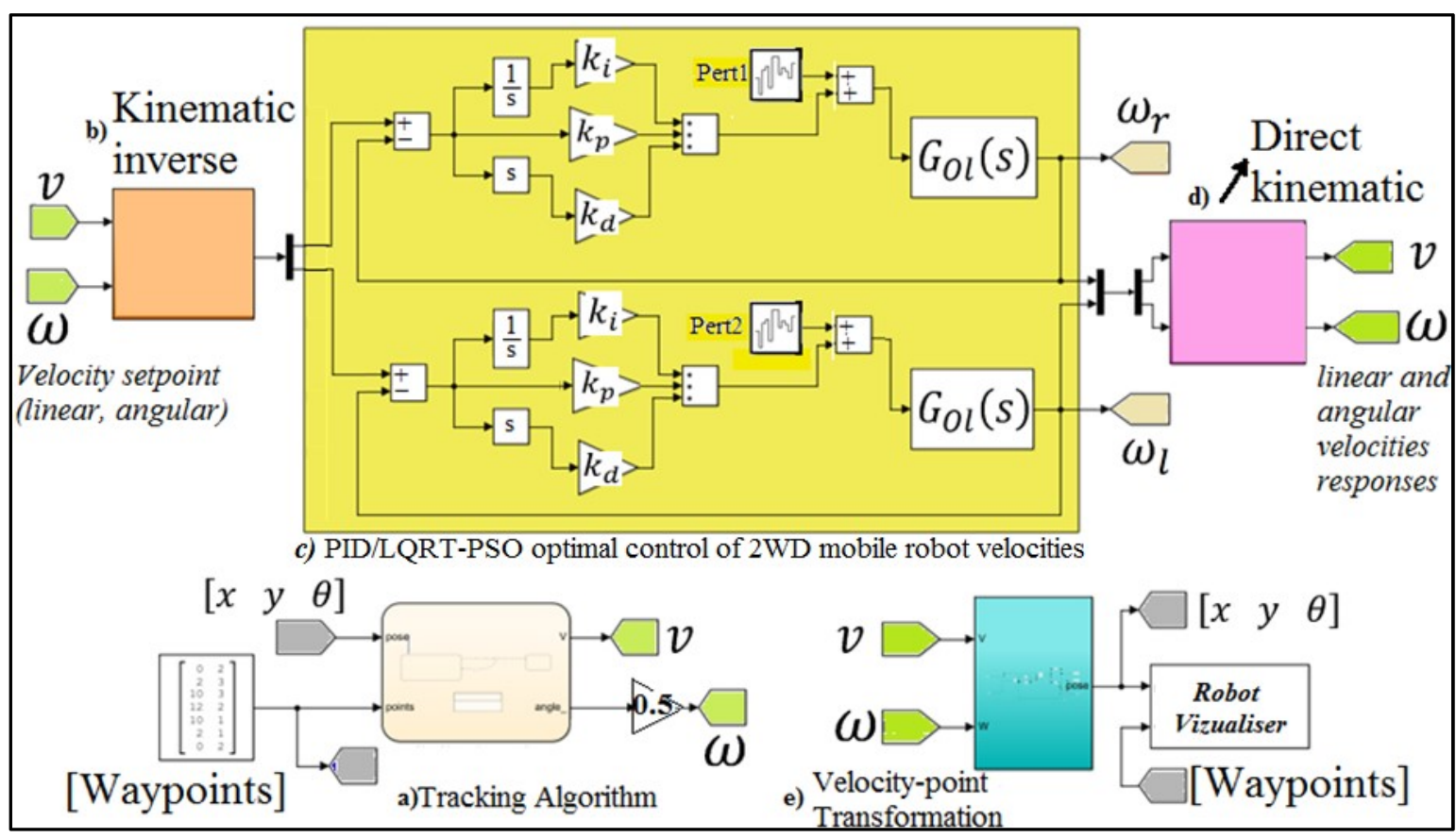

Fig. 5. Simulink model of the proposed PID/LQRT-PSO control system for 2 WDmobile robots 


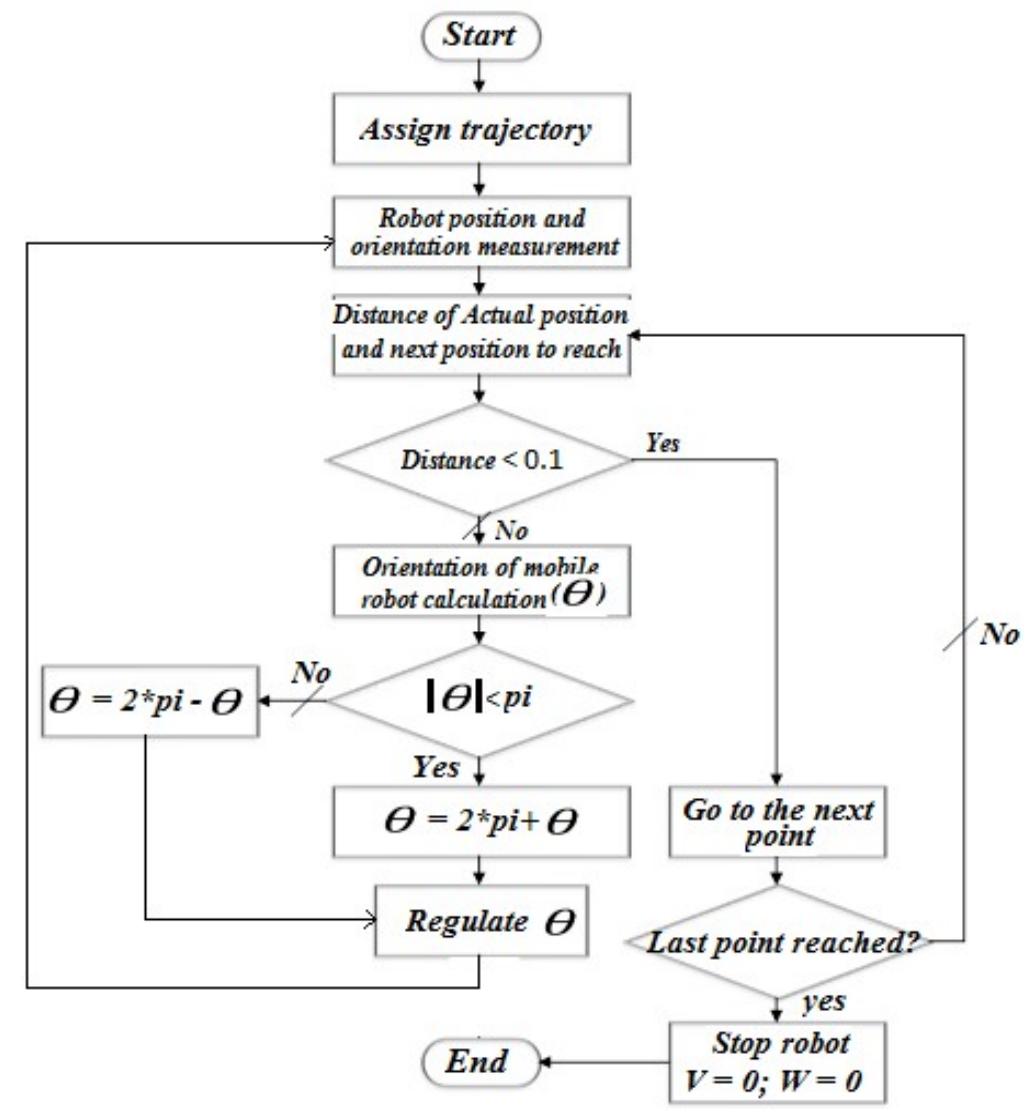

Fig. 6. Tracking trajectory flowchart 


\section{RESULTS AND DISCUSSION}

\subsection{Simulation of angular velocity for LQRT-PSO control subsystem}

Since both servomechanisms of the 2WD robot are similar, Table 2shows initial parameters of PSO algorithm, applied to the angular velocity response of a single servomechanism. The resulting LQRT-PSO parameters are outlined by eq.(24). On the other hand, the set of LQRT-TEC parameters is given by eq.(25).

Table 2. Initial parameters of PSO algorithm

\begin{tabular}{|c|c|}
\hline Parameters & Values \\
\hline $\begin{array}{l}\text { Number of swarm } \\
\text { particle }\end{array}$ & 50 \\
\hline Maximum iteration & 200 \\
\hline $\begin{array}{l}\text { Coefficients } \\
\text { acceleration } c_{1}, c_{2}\end{array}$ & 1.95 and 1.85 \\
\hline $\begin{array}{l}\text { Number of variables } \\
\left(q_{1}, q_{2}, q_{3}, r\right)\end{array}$ & 4 \\
\hline $\begin{array}{l}\text { Minimum variable } \\
\text { vector }\end{array}$ & {$\left[\begin{array}{llll}1 & 1 & 1 & 1\end{array}\right]$} \\
\hline $\begin{array}{l}\text { Maximum variable } \\
\text { vector }\end{array}$ & {$\left[\begin{array}{llll}230 & 230 & 230 & 230\end{array}\right]$} \\
\hline
\end{tabular}

$$
\left\{\begin{array}{c}
Q_{P S O}=\left[\begin{array}{ccc}
50.8884 & 0 & 0 \\
0 & 113.7679 & 0 \\
0 & 0 & 75.8359
\end{array}\right] \\
R_{P S O}=43.1824 \\
K_{\infty L Q R T-P S O}=\left[\begin{array}{lll}
1.0247 & 2.1641 & 1.3252
\end{array}\right] \\
P_{\infty L Q R T-P S O}=\left[\begin{array}{lll}
11.0617 & 23.3626 & 14.3064 \\
23.3626 & 74.3239 & 45.7719 \\
14.3064 & 45.7719 & 65.0430
\end{array}\right]
\end{array}\right.
$$

$$
\left\{\begin{array}{c}
Q_{T E C}=\left[\begin{array}{ccc}
10 & 0 & 0 \\
0 & 13 & 0 \\
0 & 0 & 1
\end{array}\right] \\
R_{T E C}=1 \\
K_{T E C}=\left[\begin{array}{lll}
2.5169 & 3.6408 & 1.0000
\end{array}\right] \\
P_{\infty T E C}=\left[\begin{array}{lll}
0.6292 & 0.9102 & 0.2500 \\
0.9102 & 4.6147 & 1.1729 \\
0.2500 & 1.1729 & 1.7015
\end{array}\right]
\end{array}\right.
$$

Figure 7 illustrates the convergence zone where we have the best cost of the fitness function as earlier defined by eq.(9). The Simulink block diagram of the LQRT-PSO controller is illustrated on Figure 8.It appears to be relevant for virtual simulation needs of the LQRT-PSO speed control subsystem.

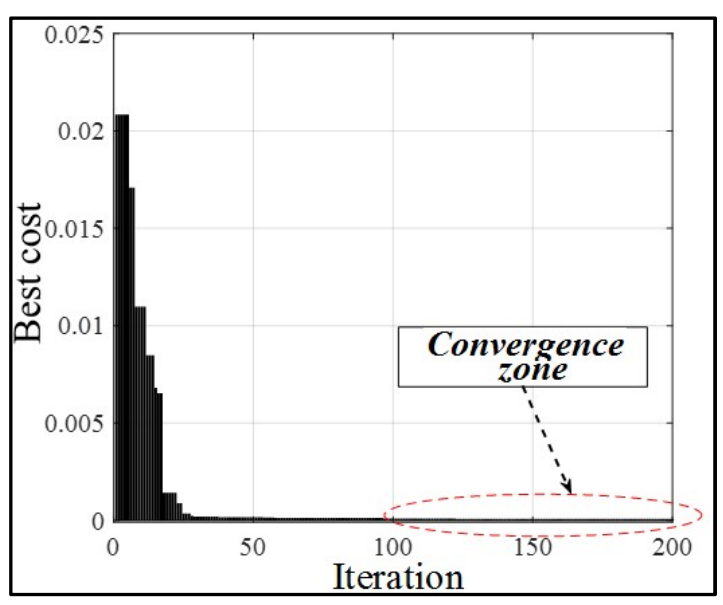

Fig. 7. Convergence zone of Best cost 


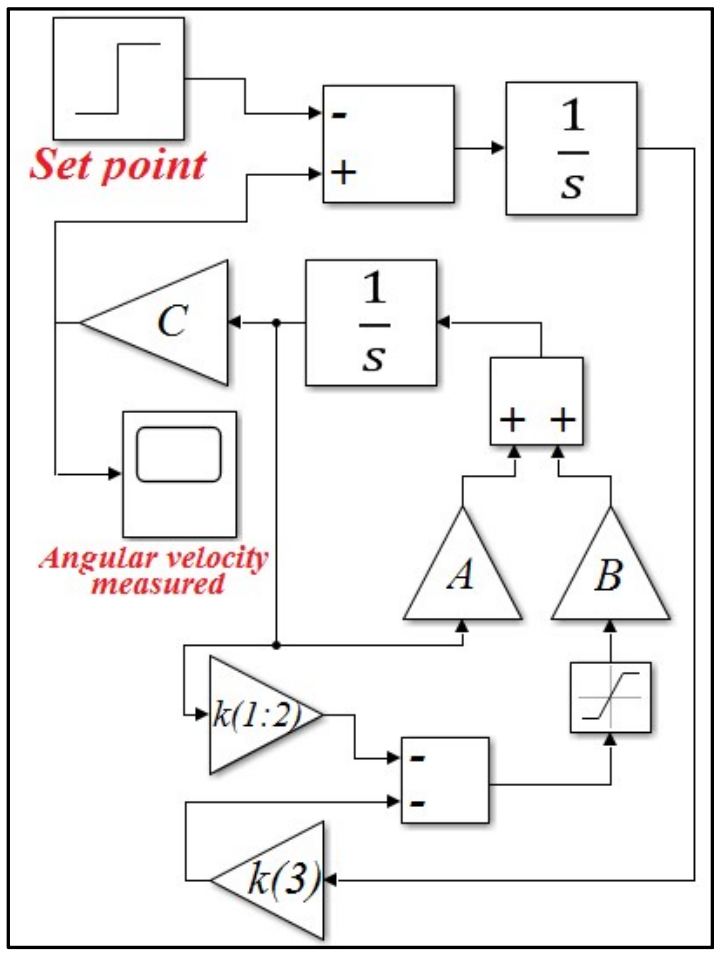

Fig. 8. Simulink block scheme of LQRT-PSO control subsystem

Simulation results with LQRT-PSO and LQRT-TEC are illustrated in Figures 9 and 10, and in Table 3. From these results, it is clear as expected, that better closed loop settling times are achieved for LQRT-PSO. Figure 10 illustrates the control voltage response obtained. It's clear that the Kalman gain used in the LQRT-PSO structure, is related to equivalent PID gains according to the linear transform eq.(17-18).

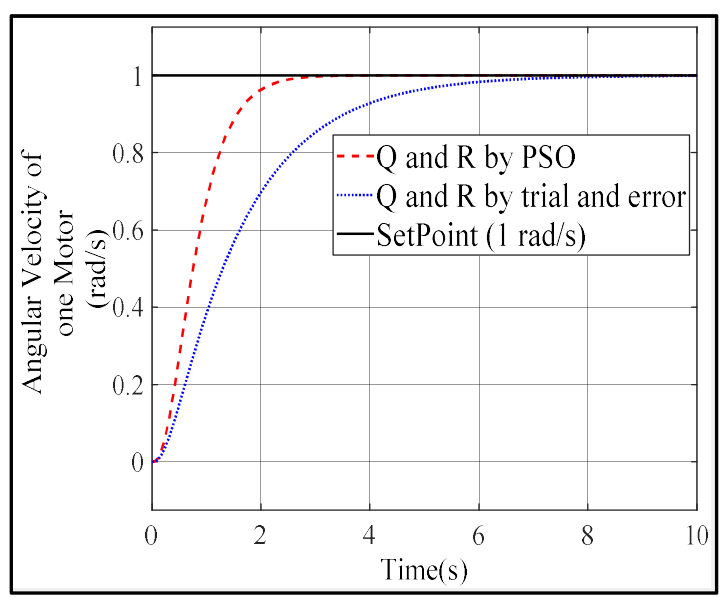

Fig. 9. Angular velocity control for LQRT-TEC and LQRT-PSO

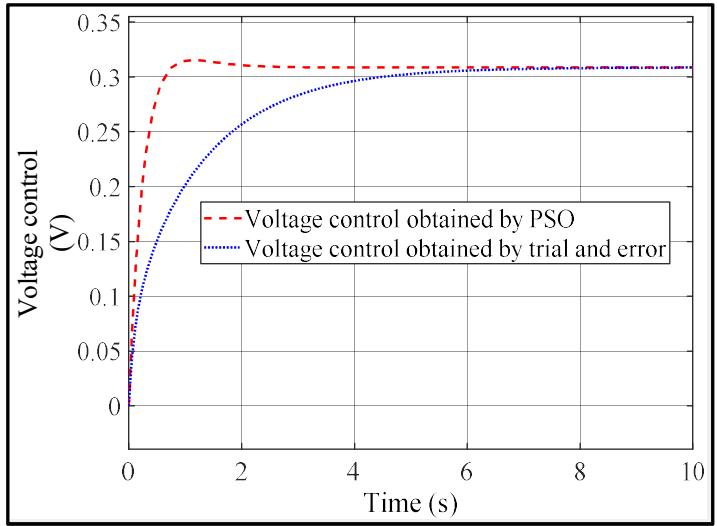

Fig. 10. Voltage control for LQRT-TEC and LQRT-PSO

Table 3. Performance index obtained

\begin{tabular}{ccc}
\cline { 2 - 3 } & LQRT-TEC & $\begin{array}{c}\text { LQRT } \\
\text {-PSO }\end{array}$ \\
\hline $\begin{array}{c}\text { Settling } \\
\text { Time (s) } \\
\text { Overshoot } \\
\text { (\%) }\end{array}$ & 5.78 & 2.25 \\
\hline
\end{tabular}

\subsection{Simulation of angular velocity for PID/LQRT-PSO control subsystem}

In this case, the PID/LQRT-PSO control gains, i.e., $K_{p}, K_{i}$ and $K_{d}$, provided in eq.(25), are computed according to eq.(17) and eq.(18), since LQRT parameters are known.

$$
\left[\begin{array}{lll}
K_{p} & K_{i} & K_{d}
\end{array}\right]=\left[\begin{array}{lll}
0.8279 & 1.3252 & 0.0980
\end{array}\right]
$$

The simulation results obtained under PID/LQRT-PSO control architecture, without disturbance, are illustrated on Figures 11 and 12. Then the related performances are compared in Table 4. 


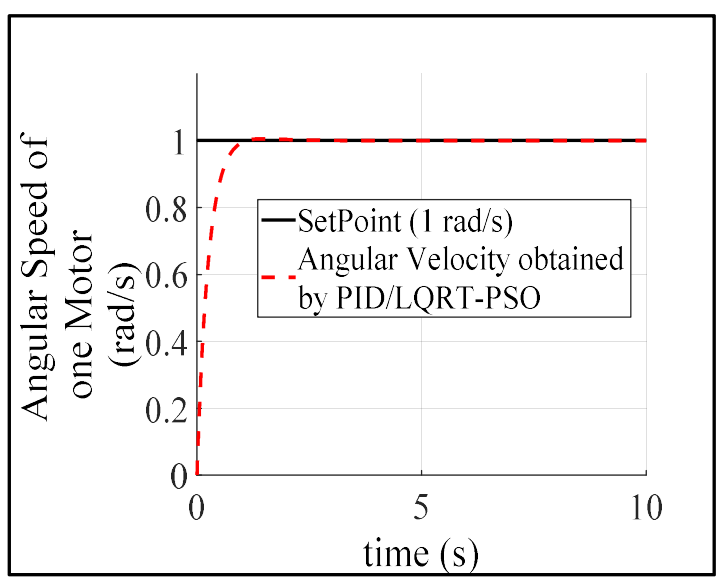

Fig. 11. Angular velocity under PID/LQRT$P S O$ control without disturbance

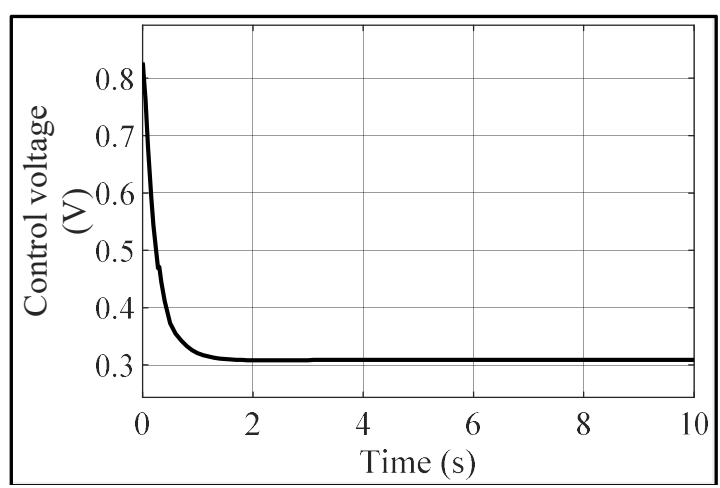

Fig. 12. Control voltage under PID/LQRTPSO control without Disturbance

Table 4. Performance of LQRT-PSO and $P I D / L Q R T-P S O$ controls

\begin{tabular}{ccc}
\cline { 2 - 3 } & LQRT-PSO & $\begin{array}{c}\text { PID/LQRT- } \\
\text { PSO }\end{array}$ \\
\hline $\begin{array}{c}\text { Settling } \\
\text { Time (s) } \\
\text { Rise time } \\
\text { (s) }\end{array}$ & 2.25 & 0.858 \\
$\begin{array}{c}\text { Overshoot } \\
\text { (\%) }\end{array}$ & 1.26 & 0.529 \\
\hline
\end{tabular}

Furthermore, to evaluate the robustness of PID/LQRT-PSO controller, white noise disturbance, with uniformly distributed within the random set[-0.06 $\mathbf{0 . 0 6}$, is added to the control input. The result are illustrated in Figures 13 and 14 , the set point tracking remains quite active over time.

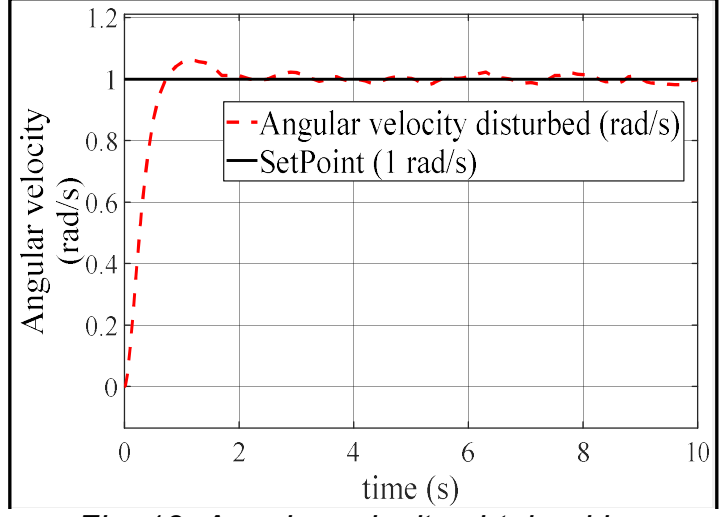

Fig. 13. Angular velocity obtained by PID/LQRT-PSO with disturbance

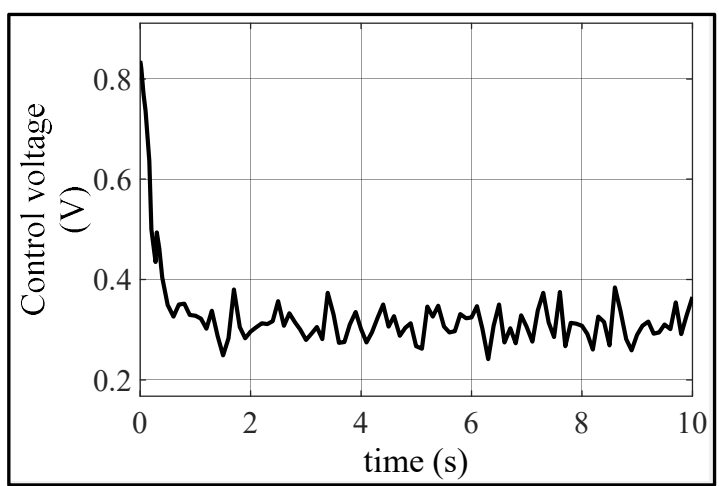

Fig. 14. Control voltage obtained by PID/LQRT-PSO under Disturbance

However, Figure 15 shows that the set point tracking behavior can be lost under very high bounds of disturbance, e.g. [-0.5 0.5].

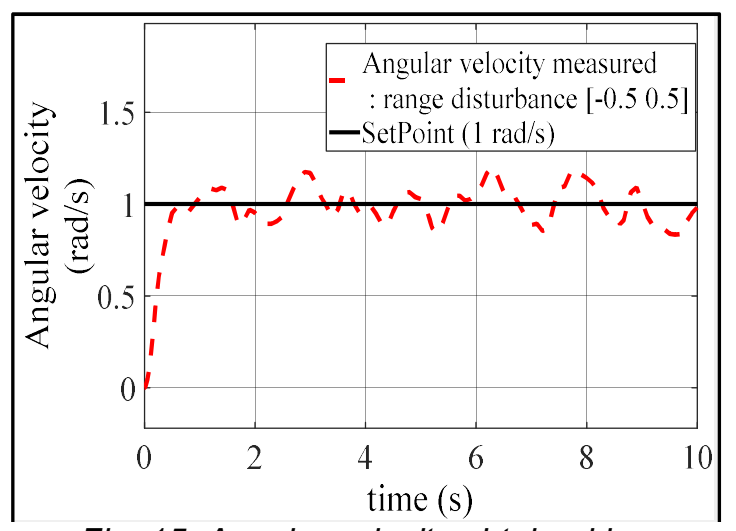

Fig. 15. Angular velocity obtained by PID/LQRT-PSO with high disturbance

However, greater number of simulation runs should be conducted, for a rigorous estimation of the robustness margin under disturbance.

\subsection{Simulation of trajectory tracking}

The trajectory tracking algorithm presented earlier in Figure 5, has been also simulated. 
The simulation results are depicted on Figures 16 and 17, for 2 types of arbitrary trajectories sets.

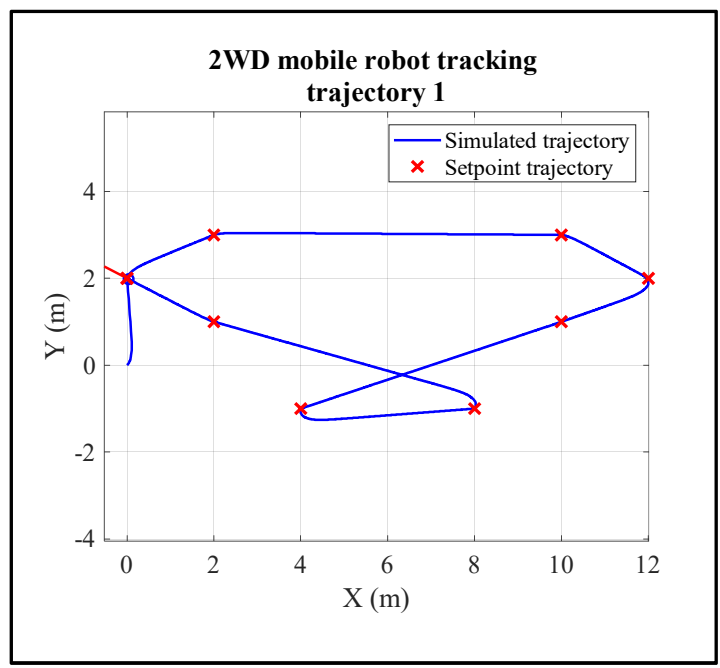

Fig. 16. Simulation of tracking trajectory 1

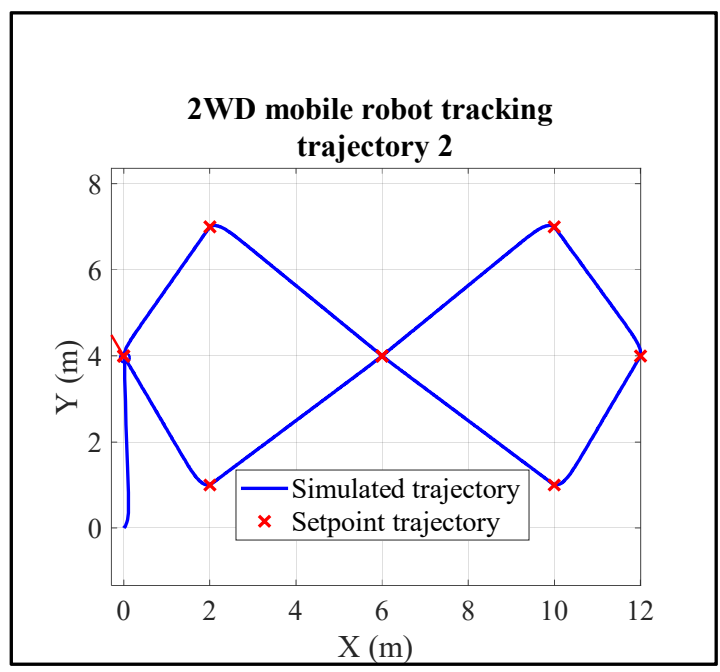

Fig. 17. Simulation of tracking trajectory 2

In figure 16, we can see the first simulation of one arbitrary trajectory which describes a cup with triangular base; however we can also see that angles taken by the 2WD mobile robot are rounded; this is due in reason of flexibility of mobile robot to move itself. When the mobile robot reaches on the next point, it decreases the velocity in order to follow the better turning angle. Otherwise, in figure 17, another arbitrary trajectory which describes approximately the infinite symbol is simulated. Furthermore, we can note that, in the two cases of figures 16 and 17, the 2WD mobile robot follows the predefined trajectories with better performances as summarized earlier in table 4.

\section{CONCLUSION}

This pioneering paper has proved the virtual feasibility of PID/LQRT-PSO control systems for 2WD mobile robots. It offers higher quality compared to popular PID/LQRT-TEC schemes. In addition, on the basis of two relevant case studies, it has been also shown that, a suitable algorithm operating downstream the PID/LQRT-PSO controller, can be used might to rigorously solving .However, it would be relevant to estimate in depth, the admissible lower and upper robustness bounds, of the proposed PID/LQRT-PSO control scheme for 2WD mobile robot. Furthermore, a digital implementation of the proposed optimal control scheme, using SOC (System On Chip) technology, will be a significant contribution for future research works.

\section{REFERENCES}

[1] H. Maghfiroh, M. Nizam, and S. Praptodiyono, "PID optimal control to reduce energy consumption in DCdrive system," International Journal of Power Electronics and Drive System, vol. 11, no. 4, pp. 2164-2172, 2020, doi: 10.11591/ijpeds.v11.i4.pp21642172.

[2] P. W. Oliveira, G. A. Barreto, and G. A. P. Thé, "A General Framework for Optimal Tuning of PID-like Controllers," Journal of Intelligent and Robotic Systems, no. 99, pp. 467486, 2020, doi: https://doi.org/10.1007/s10846-01901121-y.

[3] Y. Zennir, S. Grief, and E. Mechhoud, "Straddle Robot Design and control with a PID controller optimized by PSO algorithms," Algerian Journal of Sgnals and Sysems, vol. 5, no. 2, pp. 142-147, 2020.

[4] S. Swadi, A. I. Majeed, and M. A. Shuriji, "Design and Simulation of Robotic Arm PD Controller Based on PSO," Thi_Qar University Journal for Engineering Sciences, no. March 2018, pp. 1-7, 2017.

[5] M. A. Sahib and B. S. Ahmed, "A new multiobjective performance criterion used in PID tuning optimization algorithms," Journal of Advanced Research, vol. 7, no. 1, pp. 125-134, 2016, doi: 10.1016/j.jare.2015.03.004.

[6] B. Riadl, H. Youcef, M. Mohammed, and M. Elarkam, "Particle Swarm Optimization for tuning a Fuzzy Supervisory Controller Parametesrs ( 
Takagi Seguno and Mamdani Engines )," Algerian Journal of Sgnals and Sysems, vol. 5, no. 2, pp. 92-97, 2020.

[7] T. Abut, M. Hüseyinoğlu, and A. Makalesi, "Modeling and Optimal Trajectory Tracking Control of Wheeled a Mobile Robot," Caucasian Journal of Science, vol. 6, no. 2, pp. 2148-6840, 2019.

[8] A. J. Moshayedi, "Trajectory Tracking of Two-Wheeled Mobile Robots, Using LQR Optimal Control Method, Based On Computational Model of KHEPERA IV," Journal of Simulation \& Analysis of Novel Technologies in Mechanical Engineering, vol. 3, no. 10, pp. 0041-0050, 2017.

[9] A. Abbasi and A. J. Moshayedi, "Trajectory tracking of two-wheeled mobile robots, using LQR optimal control method, based on computational model of KHEPERA IV," Journal of Simulation and Analysis of Novel Technologies in Mechanical Engineering, vol. 10, no. 3, pp. 41-50, 2018.

[10] J. Fang, "LQR control of mobile Robot.pdf," Mathematical Problems in Engineering, pp. 1-6, 2014, doi: http://dx.doi.org/10/1155/2014/729095

[11] A. I. Abdulla, I. K. Mohammed, and A. M. Jasim, "Roll Control System Design Using Auto Tuning LQR Technique," International Journal of Engineering and Innovative Technology (IJEIT), vol. 7, no. 1, pp. 10-17, 2017.

[12] P. O. Etouke, L. N. Nneme, and J. Mbihi, "An Optimal Control Scheme for a Class of Duty-Cycle Modulation Buck Choppers: Analog Design and Virtual Simulation," Journal of Electrical Engineering, Electronics, Control and Computer Science JEEECCS, vol. 6, no. 19, pp. 13-20, 2020.

[13] A. Al-Mahturi and H. Wahid, "Optimal Tuning of Linear Quadratic Regulator Controller Using a Particle Swarm Optimization for Two-Rotor Aerodynamical System," International Journal of Electronics and Communication Engineering, vol. 11, no. 2, pp. 196-202, 2017, doi: 10.5281/zenodo.1128899.

[14] S. Howimanporn, S. Thanok, S. Chookaew, and W. Sootkaneung,
"Design and implementation of PSO based LQR control for inverted pendulum through PLC," SII 2016 2016 IEEE/SICE International Symposium on System Integration, no. December, pp. 664-669, 2017, doi: 10.1109/SII.2016.7844075.

[15] Y. Zhang, Y. Li, Y. Liu, and G. Yi, "Control of Cricket System Using LQR Controller Optimized by Particle Swarm Optimization," Journal of Physics: Conference Series, vol. 1670, no. 1, pp. 0-6, 2020, doi: 10.1088/1742-6596/1670/1/012016.

[16] M. R. Mortazavi and A. Naghash, "Pitch and flight path controller design for F-16 aircraft using combination of LQR and EA techniques," Proceedings of the Institution of Mechanical Engineers, Part G: Journal of Aerospace Engineering, vol. 232, no. 10, pp. 1831-1843, 2018, doi: 10.1177/0954410017703144.

[17] J. Mbihi, "Synthesis and Computeraided Simulation of Digital Feedback Control Systems," in Analog Automation and Digital Feedback Control Techniques, ISTE and W., London: ISTE, John Wiley \& Sons, 2018, p. pp.39-58.

[18] M. S. Calovic and N. M. Cuk, "Proportional-Integral Derivative Realisation of Optimal LinearQuadratic Regulators.," Proceedings of the Institution of Electrical Engineers, vol. 121, no. 11, pp. 14411443, 1974, doi: 10.1049/piee.1974.0302.

[19] S. Mukhopadhyay, "P.I.D. equivalent of optimal regulator," Electronics Letters, vol. 14, no. 25, pp. 821-822, 1978.

[20] J. B. He, Q. G. Wang, and T. H. Lee, "PI/PID controller tuning via LQR approach," in Proceedings of the IEEE Conference on Decision and Control, 1998, vol. 1, no. December, pp. 1177-1182, doi: 10.1109/cdc.1998.760858.

[21] J. B. He, Q. G. Wang, and T. H. Lee, "PI/PID controller tuning via LQR approach," Chemical Engineering Science, vol. 55, no. 13, pp. 24292439 , 2000, doi: 10.1016/S00092509(99)00512-6.

[22] K. H. Reddy and P. Ramanathan, "A new feedback gain matrix based LQR $\mathrm{PI}$ controller for integrator time delay 
process," Gazi University Journal of Science, vol. 30, no. 4, pp. 232-251, 2017.

[23] O. Saleem and M. Rizwan, "Performance optimization of LQRbased PID controller for DC-DC buck converter via iterative-learning-tuning of state-weighting matrix," International Journal of Numerical Modelling: Electronic Networks, Devices and Fields, vol. 32, no. 3, pp. 1-17, 2019, doi: 10.1002/jnm.2572.

[24] J. Mbihi, "Experimental Modeling Approach of Dynamic Processes," in Analog Automation and Digital Feedback Control Techniques, ISTE and W., London: ISTE and John Wiley\&Sons.

[25] Xin-SheYang, "Particle Swarm Optimization," in Engineering Optimization: An Introduction with Metaheuristic Applications, Cambridge, United Kingdom: John Wiley \& Sons, 2010, pp. 203-211.

[26] S. Noga, "Kinematics and dynamics of some selected two-wheeled mobile robots," Archives of Civil and Mechanical Engineering, vol. 6, no. 3, pp. 55-70, 2006, doi: 10.1016/S16449665(12)60241-6. 